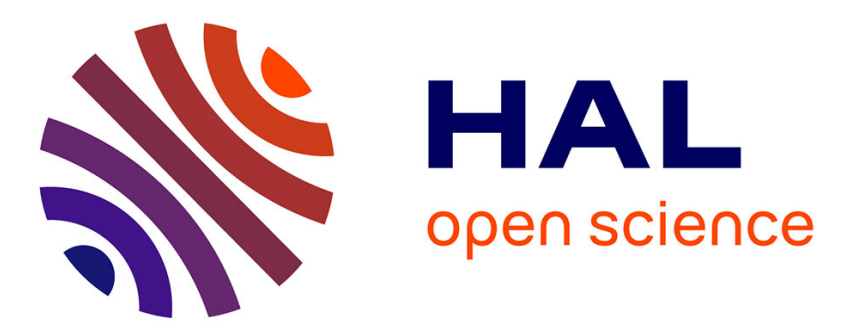

\title{
Solutions quasi analytiques de l'équation d'absorption de l'eau par les sols non saturés. II. - Problème inverse: détermination de la diffusivité capillaire
}

Michel Vauclin, Randel Haverkamp

\section{- To cite this version:}

Michel Vauclin, Randel Haverkamp. Solutions quasi analytiques de l'équation d'absorption de l'eau par les sols non saturés. II. - Problème inverse : détermination de la diffusivité capillaire. Agronomie, 1985, 5 (7), pp.607-611. hal-00884790

\author{
HAL Id: hal-00884790 \\ https://hal.science/hal-00884790
}

Submitted on 1 Jan 1985

HAL is a multi-disciplinary open access archive for the deposit and dissemination of scientific research documents, whether they are published or not. The documents may come from teaching and research institutions in France or abroad, or from public or private research centers.
L'archive ouverte pluridisciplinaire HAL, est destinée au dépôt et à la diffusion de documents scientifiques de niveau recherche, publiés ou non, émanant des établissements d'enseignement et de recherche français ou étrangers, des laboratoires publics ou privés. 


\section{Solutions quasi analytiques de l'équation d'absorp- tion de l'eau par les sols non saturés. II. - Pro- blème inverse : détermination de la diffusivité capillaire}

Michel VAUCLIN \& Randel HAVERKAMP

Institut de Mécanique de Grenoble, L.A. C.N.R.S., $n^{\circ}$ 6, B.P. 68, F 38402 Saint-Martin-d'Hères Cedex

L'analyse théorique précédente, portant sur la recherche de solutions quasi analytiques approchées de l'équation de diffusion non linéaire est appliquée à la résolution du problème inverse : déterminer la diffusivité capillaire d'un sol à partir de simples mesures de la loi d'adsorption et de l'évolution temporelle du front d'humidification. La méthode proposée permet également de quantifier les incertitudes associées à la détermination de la diffusivité dans le cas d'une fonction exponentielle de l'humidité.

Mots clés additionnels : Relation flux-concentration, sorptivité capillaire, front d'humidification, analyse d'erreurs. problem : determination of capillary diffusivity

The theoretical analysis developed in a previous paper, involving approximate quasi-analytical solutions of the non-linear diffusion equation, was applied to the inverse problem : determination of soil capillary diffusivity from simple measurements of both the adsorption law and the advance of the wetting front with time. The method was also shown to permit estimation of the error associated with any calculated diffusivity value.

Additional key words : Flux-concentration relation, capillary sorptivity, wetting front, error analysis.

\section{INTRODUCTION}

L'application de la théorie des écoulements en milieux poreux non saturés à la physique du sol se heurte bien souvent à la détermination expérimentale des propriétés hydrodynamiques des sols. Les méthodes habituellement utilisées tant au laboratoire (BRUCE \& KlUTE, 1956 ; Miller \& ElRICK, 1958 ; RIJTEMA, 1959 ; KUNZE \& KIRKHAM, 1962 ; YOUNGS, 1964 ; WATSON, 1966) que sur le terrain (ROSE et al., 1965 ; Hillel et al., 1972 ; VACHAUD et al., 1978) sont lourdes et bien souvent coûteuses à mettre en œuvre. A partir de l'analyse théorique présentée dans la $1^{\text {re }}$ partie de cet article (VAUCLIN \& HAVERKAMP, 1985), on propose une méthode simple de détermination de la diffusivité capillaire fondée sur la détermination de la sorptivité et la mesure de l'avancement $\mathrm{du}$ front d'humidification.

\section{THÉORIE}

L'analyse effectuée précédemment a montré qu'en introduisant la relation flux-concentration, la solution générale adimensionnelle du problème d'absorption s'exprime par les 2 équations suivantes :

$$
\begin{gathered}
\phi(\theta)=\frac{2}{S} \int_{\theta}^{1} \frac{D(\bar{\theta})}{F(\bar{\theta})} d \bar{\theta} \\
S^{2}=2 \int_{0}^{1} \theta \frac{D(\theta)}{F(\bar{\theta})} d \theta
\end{gathered}
$$

où $\phi(\theta)$ et $\mathrm{S}$ sont respectivement la variable de Boltzmann et la sorptivité capillaire réduites telles que les valeurs dimensionnelles sont données par : 


$$
\begin{gathered}
\phi^{*}\left(\theta^{*}\right)=\sqrt{\mathrm{D}_{\mathrm{r}}^{*}} \phi(\theta) \\
\mathrm{S}^{*}=\mathrm{S}\left(\theta_{\mathrm{S}}^{*}-\theta_{\mathrm{n}}^{*}\right) \sqrt{\mathrm{D}_{\mathrm{r}}^{*}}
\end{gathered}
$$

où $D_{r}^{*}$ est une valeur de référence de la diffusivité capillaire telle que $D^{*}(\theta)=D_{r}^{*} D(\theta)$.

$\theta_{\mathrm{n}}^{*}$ et $\theta_{\mathrm{S}}^{*}$ sont les teneurs volumiques en eau initiale et à saturation naturelle. On rappelle que $\theta$ est définie par :

$$
\theta=\left(\theta^{*}-\theta_{\mathrm{n}}^{*}\right) /\left(\theta_{\mathrm{s}}^{*}-\theta_{\mathrm{n}}^{*}\right)
$$

Si l'on suppose que la diffusivité capillaire est correctement décrite (GARDNER \& MAYHUGH, 1958 ; REICHARDT et al., 1972 ; MILLER \& BRESLER, 1977) par la relation exponentielle suivante :

$$
D^{*}(\theta)=D_{r}^{*} \exp \beta \theta
$$

la relation (4) donne alors :

$$
D^{*}(\theta)=\frac{S^{* 2}}{S^{2}\left(\theta_{S}^{*}-\theta_{n}^{*}\right)^{2}} \exp \beta \theta
$$

avec :

$$
\mathrm{S}^{2}=2 \int_{1}^{1} \frac{\theta \exp \beta \theta}{\mathrm{F}(\theta)} \mathrm{d} \theta
$$

La détermination de la diffusivité capillaire selon l'équation (6) nécessite donc, outre la connaissance préalable de $F(\theta)$, la mesure de la sorptivité capillaire $\mathrm{S}^{*}$, des teneurs en eau $\theta_{\mathrm{S}}^{*}$ et $\theta_{\mathrm{n}}^{*}$ et une estimation de $\beta$. Alors que REICHARDT et al. (1972) suggèrent que $\beta=8$ puisse être considérée comme une valeur universelle pour tous les sols; d'autres résultats (VAUCLIN, 1971 : CLOTHIER \& WHITE, 1981) semblent remettre en cause cette valeur. Il apparaît donc la nécessité de prédire $\beta$. Compte tenu du caractère exponentiel de la diffusivité, ce paramètre explique, en grande partie, la forme des profils d'infiltration. En effet, pour le milieu linéaire $\beta=0$ et le milieu de Dirac correspond à $\beta \rightarrow \infty$. En pratique, l'allure des profils hydriques peut être analysée par leur facteur de forme défini par :

$$
\alpha=\frac{\mathrm{S}}{\phi_{\mathrm{f}}}=\frac{\mathrm{S}^{*}}{\left(\theta_{\mathrm{S}}^{*}-\theta_{\mathrm{n}}^{*}\right) \phi_{\mathrm{f}}^{*}}
$$

où $\phi_{\mathrm{f}}$ est la valeur de $\phi(\theta)$ au front d'infiltration $\theta_{\mathrm{f}}$. Compte tenu de la relation (5), les équations (1) et (2) donnent alors :

$$
\alpha=\frac{\int_{0}^{1} \frac{\theta \exp \beta \theta}{F(\theta)} d \theta}{\int_{\theta_{\mathrm{f}}}^{1} \frac{\exp \beta \theta}{\mathrm{F}(\theta)} \mathrm{d} \theta}
$$

Pour une fonction $F(\theta)$ prescrite, l'équation (9) donne une relation fonctionnelle entre $\beta$ et le facteur de forme des profils $\alpha$ qui peut être déterminé par la mesure simultanée de $S^{*}$ et de l'avancement du front d'infiltration dans le temps $\phi_{\mathrm{f}}^{*}=\mathrm{x}^{*}\left(\theta_{\mathrm{f}}\right) \mathrm{t}^{*-1 / 2}$.
Le tableau 1 et la figure 1 donnent la relation entre $\alpha$ et $\beta$ obtenue par l'équation (9) avec $\theta_{\mathrm{f}}=0,01$. Les calculs ont été effectués avec les 2 expressions $\mathrm{F}(\theta)=\theta^{1 / 2}$ et $\mathrm{F}(\theta)=2 \theta /(\theta+1)$ considérées comme étant les plus précises (VAUCLIN \& HAVERKAMP, 1985).

La détermination de la diffusivité capillaire par l'équation (6) peut donc s'effectuer de la manière suivante :

- la mesure simultanée de la sorptivité et de l'avancement $\mathrm{du}$ front d'infiltration dans le temps, permet de calculer par l'équation (8) le facteur de forme $\alpha$, pour des conditions initiales $\left(\theta_{n}^{*}\right)$ et aux limites $\left(\theta_{\mathrm{S}}^{*}\right)$ déterminées ;

- la figure 1 donne alors la valeur du paramètre $\beta$; de $S^{2}$.

- l'équation (7) fournit la valeur correspondante

\begin{tabular}{|c|c|c|c|c|}
\hline \multirow{2}{*}{$\beta$} & \multicolumn{2}{|c|}{$F(\theta)=\theta^{1 / 2}$} & \multicolumn{2}{|c|}{$F(\theta)=2 \theta /(\theta+1)$} \\
\hline & $S^{2}$ & $\alpha$ & $S^{2}$ & $\alpha$ \\
\hline 0 & 1,333 & 0,3700 & 1,500 & 0,2682 \\
\hline 1 & 2,511 & 0,4608 & 2,718 & 0,3567 \\
\hline 2 & 5,025 & 0,5547 & 5,292 & 0,4620 \\
\hline 3 & 10,576 & 0,6415 & 10,936 & 0,5701 \\
\hline 4 & 23,185 & 0,7134 & 23,699 & 0,6653 \\
\hline 5 & 52,507 & 0,7689 & 53,268 & 0,7396 \\
\hline 6 & $1,219.10^{2}$ & 0,8099 & $1,231.10^{2}$ & 0,7932 \\
\hline 7 & $2,887 \cdot 10^{2}$ & 0,8401 & $2,908 \cdot 10^{2}$ & 0,8309 \\
\hline 8 & $6,949 \cdot 10^{2}$ & 0,8625 & $6,985 \cdot 10^{2}$ & 0,8574 \\
\hline 9 & $1,694 \cdot 10^{3}$ & 0,8795 & $1,701.10^{3}$ & 0,8766 \\
\hline 10 & $4,184 \cdot 10^{3}$ & 0,8954 & $4,185 \cdot 10^{3}$ & 0,8931 \\
\hline 11 & $1,036.10^{4}$ & 0,9034 & $1,039 \cdot 10^{4}$ & 0,9023 \\
\hline 12 & $2,594 \cdot 10^{4}$ & 0,9120 & $2,599.10^{4}$ & 0,9114 \\
\hline 15 & $4,209 \cdot 10^{5}$ & 0,930 & $4,213.10^{5}$ & 0,9303 \\
\hline 20 & $4,728 \cdot 10^{7}$ & 0,948 & $4,730.10^{7}$ & 0,9485 \\
\hline$\infty$ & $\infty$ & 1 & $\infty$ & 1 \\
\hline
\end{tabular}

\section{TABLEAU}

Relations entre $S^{2}, \alpha$ et $\beta$ calculées respectivement par les équations (7) et (9) avec $F(\theta)=\theta^{1 / 2}$ et $F(\theta)=2 \theta /(\theta+1)$. $\theta_{f}=0,01$.

Dependence of $S^{2}$ and $\alpha$ on $\beta$ calculated by Eqs (7) and (9) respectively for $F(\theta)=\theta^{1 / 2}$ and $F(\theta)=2 \theta /(0+1)$. $\theta_{f}=0.01$

\section{EXEMPLE D'APPLICATION}

\section{A. Détermination de la diffusivité}

On se propose ici d'appliquer cette méthode au sable "Mont-Cenis » qui a fait l'objet d'une étude détaillée en laboratoire (VACHAUD, 1968). Rappelons brièvement que l'auteur a analysé la cinétique d'absorption horizontale d'eau dans une colonne de sable à une teneur en eau initiale $\theta_{\mathrm{n}}^{*}=0,019 \mathrm{~cm}^{3} / \mathrm{cm}^{3}$. La saturation naturelle est $\theta_{\mathrm{S}}^{*}=0,442 \mathrm{~cm}^{3} / \mathrm{cm}^{3}$. Les évolutions dans le temps de la lame d'eau absorbée, $I^{*}=S^{*} \sqrt{t^{*}}$ et de l'avancement du front d'humectation $\mathrm{x}_{\mathrm{f}}^{*}=\theta_{\mathrm{f}}^{*} \sqrt{\mathrm{t}^{*}}$, ont été mesurées (cf. VACHAUD, 


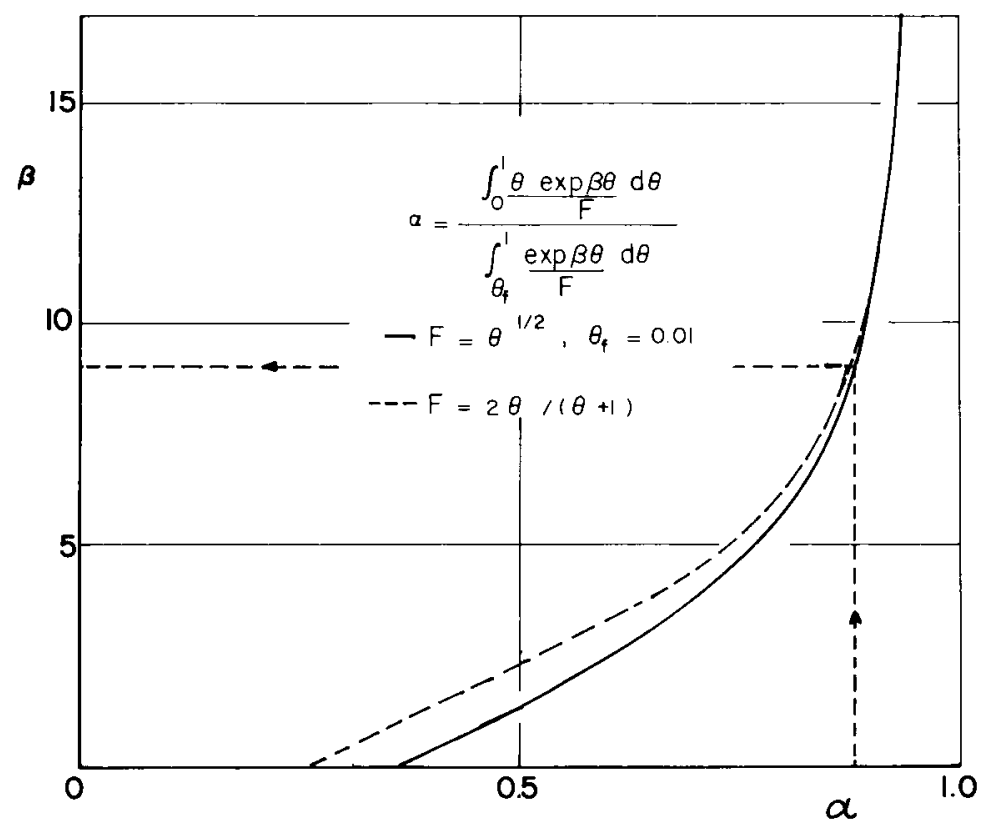

Figure 1

Relation entre $\alpha$ et $\beta$ calculée avec $F(\theta)=0^{1 / 2}$ et $F(\theta)=2 \theta /$ $(\theta+1)$ pour $\theta_{f}=0,01$ (Eq. 9).

1968, p. 77 et 79). L'analyse des résultats présentés conduit aux relations suivantes :

$$
\begin{array}{ll}
I^{*}=0,3464 \sqrt{t^{*}}+0,002 & (r=0,9996) \\
x_{f}^{*}=0,9295 \sqrt{t^{*}}-0,0396 & (r=0,9998)
\end{array}
$$

où $\mathrm{I}^{*}$ et $\mathrm{x}_{\mathrm{f}}^{*}$ sont en $\mathrm{cm}, \mathrm{t}^{*}$ en $\mathrm{mn}$ et $\mathrm{r}$ est le coefficient de corrélation.

Cela donne respectivement $\mathrm{S}^{*}=0,3464 \mathrm{~cm} \cdot \mathrm{mn}^{-1 / 2}$ et $\phi_{\mathrm{f}}^{*}=0,9295 \mathrm{~cm} \cdot \mathrm{mn}^{-1 / 2}$. On notera que les ordonnées à l'origine des relations $I^{*}\left(\sqrt{t^{*}}\right)$ et $x_{f}^{*}\left(\sqrt{t^{*}}\right)$, bien que très faibles, rendent compte de l'impédance de la plaque poreuse utilisée pour réaliser l'essai d'absorption.

L'équation (8) donne $\alpha=0,88$. Selon le choix de la relation $F(\theta)$, on obtient les résultats suivants :

- pour $F(\theta)=\theta^{1 / 2} \beta=9$ (fig. 1); $S^{2}=1693,8$ (eq. 6) soit $D^{*}(\theta)=3,96 \cdot 10^{-4} \exp 9 \theta\left(\mathrm{cm}^{2} \mathrm{mn}^{-1}\right)$

- pour $\mathrm{F}(\theta)=2 \theta /(\theta+1) \quad \beta=9,2$ (fig. 1); $\mathrm{S}^{2}=2034,5$ (eq 6) soit $\mathrm{D}^{*}(\theta)=3,30 \cdot 10^{-4} \exp 9,2 \theta$ $\left(\mathrm{cm}^{2} \cdot \mathrm{mn}^{-1}\right)$

Les relations entre la diffusivité capillaire et l'humidité ainsi estimées sont reportées figure 2 ainsi que les valeurs expérimentales données par VACHAUD (1968). On notera que ces dernières ont été obtenues par la méthode des profils instantanés, appliquée à un essai d'absorption horizontale.

\section{B. Estimation de l'erreur associée au calcul de la dif- fusivité}

Bien que l'accord entre les valeurs expérimentales et calculées de la diffusivité capillaire semble très acceptable, il nous paraît essentiel d'estimer les erreurs associées au modèle proposé. En effet, les incertitudes liées aux mesures de $\theta_{\mathrm{S}}^{*}, \theta_{\mathrm{n}}^{*}, \mathrm{~S}^{*}, \phi_{\mathrm{f}}^{*}$, d'une part, l'imprécision avec laquelle le paramètre $\beta$ est
Relationship between $\alpha$ and $\beta$ with $F(\theta)=\theta^{1 / 2}$ and $F(\theta)=2 \theta /$ $(\theta+1)$ for $\theta_{f}=0.01($ Eq. 9).

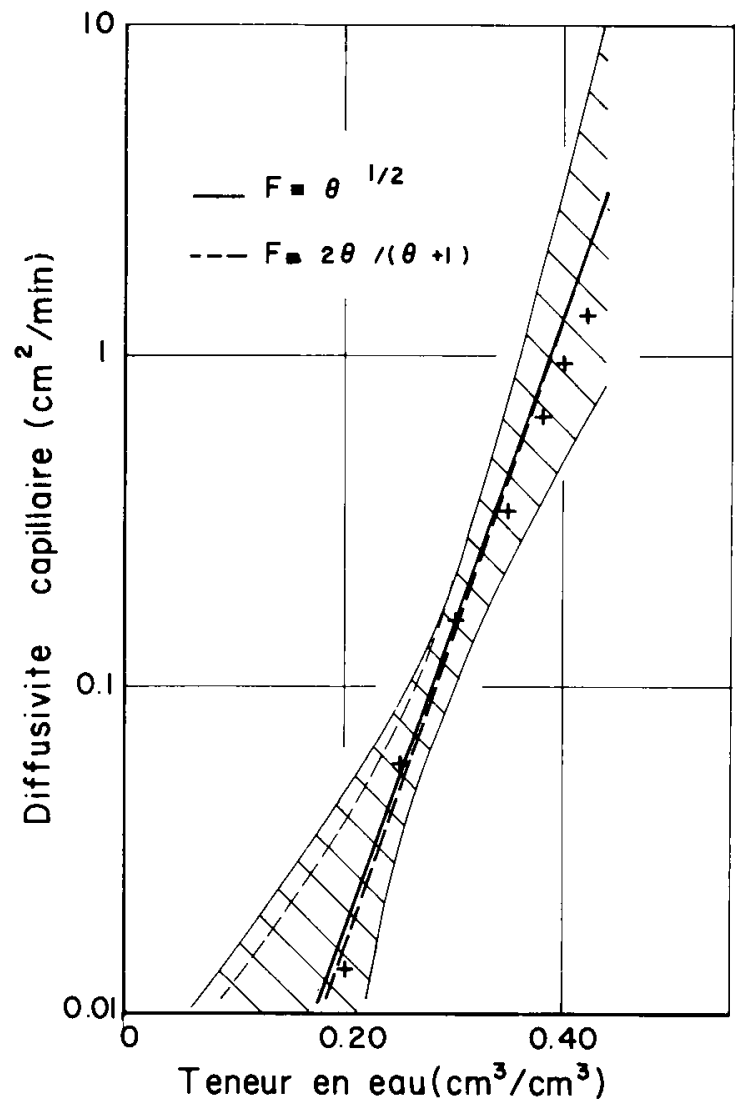

Figure 2

Diffusivité capillaire expérimentale $(+)$ et calculée $(-)$. Les zones hachurées correspondent à la bande de confiance à 68 p. 100.

Experimental (+) and calculated (-) soil water diffusivity values. Hatched areas correspond to the $68 \%$ confidence interval.

obtenu à partir du facteur de forme $\alpha$, d'autre part, vont engendrer une erreur sur l'estimation de la relation $\mathrm{D}^{*}(\theta)$. 
L'analyse d'erreur donnée en annexe montre que l'incertitude relative $\frac{\Delta D^{*}}{D^{*}}$ (eq. A10) associée à une détermination de $D^{*}$ est de la forme suivante :

$$
\begin{aligned}
\frac{\Delta D^{*}}{D^{*}} \leqslant F_{S^{*}}(\beta, \theta) \frac{\Delta S^{*}}{S^{*}}+F_{\phi_{\mathrm{f}}^{*}} & (\beta, \theta) \frac{\Delta \phi_{\mathrm{f}}^{*}}{\phi_{\mathrm{f}}^{*}}+ \\
& +\mathrm{F}_{\theta^{*}}(\beta, \theta) \frac{\Delta \theta^{*}}{\theta_{\mathrm{S}}^{*}-\theta_{\mathrm{n}}^{*}}
\end{aligned}
$$

où les coefficients $F_{S^{*}}, F_{\phi f}, F_{\theta^{*}}$ sont donnés par l'équation (A10).

L'équation (13) met clairement en évidence, les contributions respectives des incertitudes expérimentales affectant la sorptivité $\left(\Delta S^{*} / S^{*}\right)$, l'avancement du front d'humectation $\left(\Delta \phi_{\mathrm{f}}^{*} / \phi_{\mathrm{f}}^{*}\right)$ et l'humidité $\left(\Delta \theta^{*} /\left(\theta_{\mathrm{S}}^{*}-\theta_{\mathrm{n}}^{*}\right)\right)$, à l'erreur totale $\Delta D^{*} / D^{*}$. Elle montre également que cette erreur dépend du sol (par l'intermédiaire de $\beta$ ) et pour un sol donné de l'humidité $(\theta)$.

Le calcul pratique du majorant de l'erreur $\Delta D^{*} / D^{*}$ requiert la détermination de $\Delta \mathrm{S}^{*}, \Delta \phi_{\mathrm{f}}^{*}$ et $\Delta \theta^{*}$.

Les erreurs relatives sur la sorptivité et la transformée de Boltzmann peuvent être estimées à partir des écarts-types sur la pente des droites de régression $I^{*}=f\left(\sqrt{t^{*}}\right)$ et $x_{f}^{*}=g\left(\sqrt{t^{*}}\right)$ selon les formules classiques de statistique (VAUCLIN et al., 1983). Aussi dans le cas particulier précédent (eq. 10) cela donne :

et

$$
\begin{aligned}
& \sigma\left(\mathrm{S}^{*}\right)= \pm 2,94 \cdot 10^{-3} \mathrm{~cm} \cdot \mathrm{mn}^{-1 / 2} \\
& \sigma\left(\phi_{\mathrm{f}}^{*}\right)= \pm 4,51 \cdot 10^{-3} \mathrm{~cm} \cdot \mathrm{mn}^{-1 / 2}
\end{aligned}
$$

soit

$$
\frac{\Delta \mathrm{S}^{*}}{\mathrm{~S}^{*}}=\frac{\left|\sigma\left(\mathrm{S}^{*}\right)\right|}{\mathrm{S}^{*}}=8,48 \cdot 10^{-3}
$$

et

$$
\frac{\Delta \phi_{\mathrm{f}}^{*}}{\phi_{\mathrm{f}}^{*}}=\frac{\left|\sigma\left(\phi_{\mathrm{f}}^{*}\right)\right|}{\phi_{\mathrm{f}}^{*}}=4,85 \cdot 10^{-3}
$$

En supposant que les humidités volumiques sont mesurées à $\Delta \theta^{*}=0,01 \mathrm{~cm}^{3} / \mathrm{cm}^{3}$ près (VAUCLIN, 1971), les bandes assimilées à $\pm \Delta D^{*}$ et calculées selon l'équation (13) pour les 2 relations $F(\theta)$ sont reportées figure 2 . Les remarques suivantes peuvent être faites :

- le choix de la fonction $F(\theta)$ influence peu la prédiction de la diffusivité ainsi que les incertitudes associées ;

- le bon accord avec les valeurs expérimentales semble qualifier le modèle proposé pour déterminer la diffusivité capillaire ;

- la largeur de la bande de confiance est fonction de l'humidité : elle atteint un ordre de grandeur de la diffusivité au voisinage de la saturation. Cela peut paraître important. Néanmoins il convient de noter que le calcul proposé conduit à un majorant de l'erreur. De plus, les valeurs expérimentales sont également entachées d'erreurs dues aux mesures de l'humidité elle-même et à la procédure d'obtention de la diffusivité (intégration de profils hydriques après lissage, calcul graphique de pentes, etc...).

\section{CONCLUSIONS}

Une méthode simple de détermination de la diffusivité capillaire d'un sol à degré de saturation variable a été présentée. Elle est théoriquement fondée sur l'utilisation de la relation flux-concentration pour résoudre de façon quasi-analytique l'équation d'absorption d'eau par un milieu partiellement saturé. Pratiquement, elle requiert la mesure de 2 grandeurs : les lois d'absorption et d'avancement d'un front d'humectation, en fonction du temps.

Les résultats ont montré un bon accord entre valeurs expérimentales et calculées, compte tenu des incertitudes sur la mesure de ces 2 grandeurs. Il apparaît que la prédiction de la diffusivité est très peu sensible au choix de la relation flux-concentration.

Bien que la méthode ait été développée pour une relation exponentielle entre diffusivité et humidité, elle s'applique à toute autre fonctionnelle à 2 paramètres. On notera qu'elle peut également être mise en œuvre dans le cas d'un écoulement vertical, à condition de ne considérer que la phase initiale du processus. En effet, au cours de cette période (quelques minutes), les effets gravitaires sont négligeables devant les effets capillaires et l'équation du transport vertical d'eau se ramène à une équation de diffusion. Un appareil de terrain dont le principe de fonctionnement repose sur cette méthode est actuellement en cours de développement. Les résultats seront présentés ultérieurement.

Reçu le 2 septembre 1984. Accepté le 8 mars 1985.

\section{ANNEXE}

La différentiation logarithmique de l'équation (6) conduit à :

$$
\frac{d D^{*}}{D^{*}}=2 \frac{d S^{*}}{S^{*}}-2 \frac{d S}{S}-2 \frac{d\left(\theta_{S}^{*}-\theta_{n}^{*}\right)}{\theta_{S}^{*}-\theta_{n}^{*}}+\theta d \beta+\beta d \theta .
$$

L'équation (7) donne :

$$
\begin{aligned}
& \frac{\mathrm{dS}}{\mathrm{S}}=\frac{\int_{0}^{1} \frac{\theta^{2} \exp \beta \theta d \theta}{\mathrm{F}(\theta)}}{2 \int_{0}^{1} \frac{\theta \exp \beta \theta d \theta}{\mathrm{F}(\theta)}} \mathrm{d} \beta \\
& \frac{\mathrm{dS}}{\mathrm{S}}=\mathrm{T}(\beta) \mathrm{d} \beta
\end{aligned}
$$

L'équation (9) apparaît sous la forme générale ;

donc :

$$
\alpha=\frac{\mathrm{U}(\beta)}{\mathrm{V}(\beta)}
$$

$$
\text { avec : } \quad W(\beta)=\frac{U^{\prime}(\beta) V(\beta)-U(\beta) V^{\prime}(\beta)}{V^{2}(\beta)}
$$


Le calcul des dérivées $U^{\prime}(\beta)$ et $V^{\prime}(\beta)$ par rapport au paramètre $\beta$ étant trivial, la définition de facteur de forme $\alpha$ (eq. 8 ) conduit à :

$$
\frac{\mathrm{d} \alpha}{\alpha}=\frac{\mathrm{dS}}{\mathrm{S}^{*}}-\frac{\mathrm{d} \phi_{\mathrm{f}}^{*}}{\phi_{\mathrm{f}}^{*}}-\frac{\mathrm{d}\left(\theta_{\mathrm{S}}^{*}-\theta_{\mathrm{n}}^{*}\right)}{\theta_{\mathrm{S}}^{*}-\theta_{\mathrm{n}}^{*}} .
$$

Compte tenu de (A6), l'équation (A5) donne :

$$
\mathrm{d} \beta=\frac{\alpha}{\mathrm{W}(\beta)}\left\{\frac{\mathrm{d} \mathrm{S}^{*}}{\mathrm{~S}^{*}}-\frac{\mathrm{d} \phi_{\mathrm{f}}^{*}}{\phi_{\mathrm{f}}^{*}}-\frac{\mathrm{d}\left(\theta_{\mathrm{S}}^{*}-\theta_{\mathrm{n}}^{*}\right)}{\theta_{\mathrm{S}}^{*}-\theta_{\mathrm{n}}^{*}}\right\} .
$$

La définition de la teneur en eau réduite $\theta=\left(\theta^{*}-\theta_{\mathrm{n}}^{*}\right) /\left(\theta_{\mathrm{S}}^{*}-\theta_{\mathrm{n}}^{*}\right)$ permet d'écrire :

$$
d \theta=\theta\left\{\frac{d\left(\theta^{*}-\theta_{n}^{*}\right)}{\theta^{*}-\theta_{n}^{*}}-\frac{d\left(\theta_{S}^{*}-\theta_{n}^{*}\right)}{\theta_{S}^{*}-\theta_{n}^{*}}\right\}
$$

Reportant successivement les équations (A3), (A7) et (A8) dans (A1), il vient :

$$
\begin{aligned}
\frac{d D^{*}}{D^{*}} & =\left\{2+\frac{\alpha}{W(\beta)}(\theta-2 T(\beta))\right\} \frac{d S^{*}}{S^{*}}-\frac{\alpha}{W(\beta)}(\theta-2 T(\beta)) \frac{d \phi_{f}^{*}}{\phi_{f}^{*}}+ \\
& +\beta \frac{d\left(\theta^{*}-\theta_{n}^{*}\right)}{\theta_{S}^{*}-\theta_{n}^{*}}-\left\{2+\beta \theta+\frac{\alpha}{W(\beta)}(\theta-2 T(\beta))\right\} \frac{d\left(\theta_{S}^{*}-\theta_{n}^{*}\right)}{\theta_{S}^{*}-\theta_{n}^{*}}
\end{aligned}
$$

En admettant que l'incertitude absolue est sensiblement constante (VAUCLIN et al., 1983), quelle que soit sa valeur $\left(\Delta \theta^{*}=\Delta \theta_{\mathrm{S}}^{*}=\Delta \theta_{\mathrm{n}}^{*}\right)$, l'erreur relative associée à une détermination $\mathrm{D}^{*}$ est alors bornée par

$$
\begin{aligned}
\frac{\Delta \mathrm{D}^{*}}{\mathrm{D}^{*}} \leqslant \mid 2 & +\frac{\alpha}{\mathrm{W}(\beta)}(\theta-2 \mathrm{~T}(\beta))\left|\frac{\Delta \mathrm{S}^{*}}{\mathrm{~S}^{*}}+\right| \frac{\alpha}{\mathrm{W}(\beta)}(\theta-2 \mathrm{~T}(\beta)) \mid \frac{\Delta \phi_{\mathrm{f}}^{*}}{\phi_{\mathrm{f}}^{*}}+ \\
& +2\left|\beta+2+\beta \theta+\frac{\alpha}{\mathrm{W}(\beta)}(\theta-2 \mathrm{~T}(\beta))\right| \frac{\Delta \theta^{*}}{\theta_{\mathrm{S}}^{*}-\theta_{\mathrm{n}}^{*}} \quad(\mathrm{~A} 10)
\end{aligned}
$$

Les barres verticales correspondent aux valeurs absolues.

\section{RÉFÉRENCES BIBLIOGRAPHIQUES}

Bruce R. R., Klute A., 1956. The measurement of soil water diffusivity. Soil Sci. Soc. Am. Proc., 20, 458-462.

Clothier B. E., White I., 1981. Measurement of sorptivity and soil water diffusivity in the field. Soil Sci. Soc. Am. J., 45, 241-245.

Gardner W. R., Mayhugh M. S., 1958. Solutions and tests of the diffusion equation for the movement of water in soil. Soil Sci. Soc. Am. Proc., 22, 197-201.

Hillel D., Krentos V. D., Stylianou Y., 1972. Procedure and test of an internal drainage method for measuring soil hydraulic characteristics in situ. Soil Sci., 114, 395-400.

Kunze R. J., Kirkham D., 1962. Simplified accounting for membrane impedance in capillary conductivity determinations. Soil Sci. Soc. Am. Proc., 26, 421-426.

Miller R. D., Bresler E., 1977. A quick method for estimating soil water diffusivity functions. Soil Sci. Soc. Am. Proc., 41, 1021-1022.

Miller E. E., Elrick D. E., 1958. Dynamic determination of capillary conductivity extended for non-negligible membrane impedance. Soil Sci. Soc. Am. Proc., 22, 483-486.

Reichardt K. D., Nielsen D. R., Biggar J. W., 1972. Scaling of horizontal infiltration into homogeneous soils. Soil Sci. Soc. Am. Proc., 36, 241-245.

Rijtema P. E., 1959. Calculation of capillary conductivity from pressure plate outflow data with non-negligible membrane impedance. Neth. J. Agr. Sci., 7, 209-215.
Rose C. W., Stern W. R., Drummond J. E., 1965. Determination of hydraulic conductivity as a function of depth and water content for soil in situ. Aust. J. Soil Res., 3, 1-9.

Vachaud G., 1968. Contribution à l'étude des problèmes d'écoulement en milieux poreux non saturés. Thèse Doct. Sci. Univ. Grenoble, $160 \mathrm{p}$.

Vachaud G., Dancette C., Sonko S., Thony J. L., 1978. Méthodes de caractérisation hydrodynamique in situ d'un sol non saturé. Application à deux types de sol du Sénégal en vue de la détermination des termes du bilan hydrique. Ann. Agron., 29 (1), 1-36.

Vauclin M., 1971. Effets dynamiques sur la relation succion-teneur en eau lors d'écoulements en milieu non saturé. Thèse Doct.-Ing. Univ. Sci. Méd. de Grenoble, 113 p.

Vauclin M., Haverkamp R., 1985. Solutions quasi analytiques de l'équation d'absorption de l'eau par les sols non saturés. I. Analyse critique. Agronomie, 5 (7).

Vauclin M., Vieira S. R., Vachaud G., Nielsen, D. R., 1983. The use of cokriging with limited field soil observations. Soil Sci. Soc. Am. J., 47, 175-184.

Watson K. K., 1966. An instantaneous profile method for determining the hydraulic conductivity of unsaturated porous materials. Water Resour. Res., 2, 709-715.

Youngs E. G., 1964. An infiltration method of measuring the hydraulic conductivity of unsaturated porous materials. Soil Sci., $109,307-311$ 